\title{
Technetium-99m-Labelled Sulesomab (LeukoScan) in the Evaluation of Soft Tissue Infections
}

\author{
Anne-Marie Quigley Gopinath Gnanasegaran \\ Andrew J.W. Hilson \\ Department of Nuclear Medicine, Royal Free Hospital, London, UK
}

John R. Buscombe

\section{Key Words}

Technetium-99m-labelled sulesomab - Soft tissue infection • LeukoScan

\begin{abstract}
Objective: To perform a retrospective review of all patients

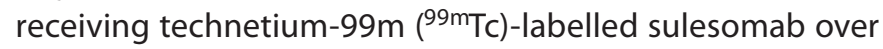
a 4-year period to determine if soft tissue infections can be accurately identified. Methods and Materials: We reviewed

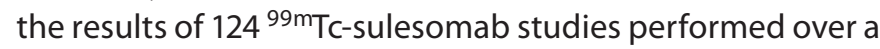
4-year period. Of these, 34 were performed for undiagnosed fever in which soft tissue infection was suspected to be the main cause. The patients' clinical notes, microbiology reports and other imaging findings were reviewed to determine the clinical outcome following the scan. The scans were regarded as being true-positives if (i) uptake correlated with the site from which fluid or tissue was obtained and which grew bacteria, and/or (ii) the site of abnormality was reported as having an infection on other imaging or (iii) there was a clinical correlation with the referring clinician's evaluation of the patient. Planar imaging was performed using standard protocols, together with single-photon emission computed tomography (if required) at 1 and $4 \mathrm{~h}$ after injection of $20-30 \mathrm{mCi}(740-1,110 \mathrm{MBq}){ }^{99 m} \mathrm{Tc}$-sulesomab. Results: Three patients were unevaluable. In the remaining
\end{abstract}

31 patients, $21{ }^{99 m}$ Tc-sulesomab studies were regarded as true-positives and 6 patients had true-negative scans. One patient had a false-positive scan (abnormal uptake with negative microbiology) and 3 had false-negative scans (infection confirmed but a negative scan). Conclusion: In sus-

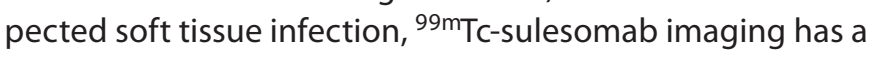
sensitivity of $88 \%$ with a specificity of $86 \%$ and overall accuracy of $87 \%$. ${ }^{99 m}$ Tc-sulesomab provides an accurate method of imaging for suspected soft tissue infection, which is also fast and convenient, as cell labelling is not required.

Copyright $\odot 2008$ S. Karger AG, Basel

\section{Introduction}

Accurate, early diagnosis of infection or inflammation is critical for optimal clinical management. Imaging by conventional modalities, namely computed tomography $(\mathrm{CT})$, ultrasound or magnetic resonance imaging, is often unrewarding in patients with pyrexia of unknown origin. In postsurgical patients, interpretation of images may be very difficult due to the presence of postoperative changes. Nuclear medicine provides a variety of radiopharmaceuticals, such as technetium- $99 \mathrm{~m}\left({ }^{99 \mathrm{~m}} \mathrm{Tc}\right)$-labelled methyldiphosphonate, gallium-67, radiolabelled monoclonal antibodies, ${ }^{99 \mathrm{~m}} \mathrm{Tc}$-labelled nanocolloids and

\section{KARGER}

Fax +41 613061234

E-Mail karger@karger.ch

www.karger.com
(C) 2008 S. Karger AG, Basel

$1011-7571 / 08 / 0176-0447 \$ 24.50 / 0$

Accessible online at:

www.karger.com/mpp
Dr. J.R. Buscombe

Nuclear Medicine Department, Royal Free Hospital

Pond Street

London NW3 2QG (UK)

Tel. +44 207830 2470, Fax +44 207472 6202, E-Mail j.buscombe@medsch.ucl.ac.uk 
white cells [labelled with either ${ }^{99 \mathrm{~m}} \mathrm{Tc}$-labelled hexamethylpropylene amine oxime (HMPAO) or ${ }^{111}$ In-labelled oxine/tropolone] that can be used to image patients with suspected infection. These agents may be used in conjunction with conventional anatomical imaging or, as is more often the case, when the latter fails to provide the answer. Functional imaging techniques may provide a reference point on which to focus further anatomical imaging, particularly when the clinical history is vague, or the signs and symptoms are rather non-specific.

Although labelled white-cell imaging is normally seen as the 'gold standard', cell preparation is time consuming, technically demanding and also requires manipulation of blood products. ${ }^{99 \mathrm{~m}} \mathrm{Tc}$-sulesomab (LeukoScan, Immunomedics, New Plains, N.J., USA) is a technetium-labelled murine monoclonal Fab' antibody fragment, the epitope of which is directed to a granulocyte surface protein (NCA-90 surface antigen). It is currently licensed in Europe for patients with suspected bone or joint infections [1]. The mechanism of action is not clear, as recent work has failed to show binding of ${ }^{99 \mathrm{~m}} \mathrm{Tc}$-sulesomab to circulating leucocytes [2]. However, studies with ${ }^{99 \mathrm{~m}} \mathrm{Tc}-$ sulesomab have shown that sensitivity, specificity and diagnostic accuracy are comparable to labelled white cells in the detection of osteomyelitis [3]. Most of the published use of ${ }^{99 \mathrm{~m}} \mathrm{Tc}$-sulesomab has been within the context of suspected bone and joint infections, and it has been reported to be useful in a number of soft tissue infections such as endocarditis, appendicitis and infected vascular graft [4].

The purpose of this study was to retrospectively assess the use of ${ }^{99 \mathrm{~m}} \mathrm{Tc}$-sulesomab in the non-orthopaedic setting and to determine its overall accuracy in identifying soft tissue infections.

\section{Patients and Methods}

\section{Patients}

A retrospective study of patients investigated for suspected non-osseous infections with ${ }^{99 \mathrm{~m}} \mathrm{Tc}$-sulesomab during a 4 -year period (from January 1, 2000, to December 31, 2004) was performed, following the introduction of the radiopharmaceutical in 2000. Patients were identified on the Nuclear Medicine and Radiology information system as having had a ${ }^{99 \mathrm{~m}} \mathrm{Tc}$-sulesomab scan. The request forms of all these patients were reviewed, and those suspected of or subsequently found to have infection within bone were excluded from analysis. A total of 124 adult patients had undergone imaging with ${ }^{99 \mathrm{~m}} \mathrm{Tc}$-sulesomab during the study period. Of these, 34 were performed for suspected soft tissue infection (20 male, 14 female; age 31-71 years). These patients' clinical notes, microbiology reports and other imaging findings were then reviewed to determine the clinical outcome of the patient.
Radiopharmaceutical and Scan Procedure

${ }^{99 m}$ Tc-sulesomab (LeukoScan) was supplied as a commercial kit and reconstituted with up to $20 \mathrm{mCi}(740 \mathrm{MBq}){ }^{99 \mathrm{~m}} \mathrm{Tc}$. It was injected intravenously within $4 \mathrm{~h}$ of reconstitution. Whole-body planar imaging was performed at 1 and $4 \mathrm{~h}$ after injection with a low-energy high-resolution collimator, table speed $8 \mathrm{~cm} / \mathrm{min}$, using a Prism 2000 XP (Phillips Picker, Eindhoven, the Netherlands). Planar images were reviewed by the nuclear medicine physician on duty at 1 and 4-6 h. Single-photon emission computed tomography (SPECT) was then performed if it was thought that the technique might be useful. Images were iteratively reconstructed using 6 iterations and ordered subset expectation maximization and filtered using a count-optimised low-pass Butterworth filter. They were then displayed in the standard 3 orthogonal planes - transverse, sagittal and coronal. Images were read by a physician of nuclear medicine. A study was recorded as positive if there was focal non-physiological accumulation of ${ }^{99 \mathrm{~m}}$ Tc-sulesomab that increased with time compared to background uptake in both image sets. The study was also regarded as positive if the uptake of ${ }^{99 \mathrm{~m}} \mathrm{Tc}$-sulesomab was markedly increased compared to expected physiological activity at that site in the first image and that level of activity was maintained in the second image set. If there was focal uptake which did not increase with time or even decreased, this was reported as negative. The results were interpreted as true-positive if the uptake correlated anatomically with the site from which infected fluid was obtained and/or with the site of abnormality reported on other imaging or if there was clinical correlation with the referring clinician's evaluation of the patient.

A true-negative result was determined as being no abnormal accumulation of ${ }^{99 \mathrm{~m}} \mathrm{Tc}$-sulesomab and subsequent negative microbiological and/or histological assessment, which, together with a minimum 6-month clinical follow-up, failed to show any evidence of infection. No patients died, though one was lost to follow-up during this 6-month period.

\section{Results}

Of 34 studies, 21 were true-positive (table 1). These included 13 patients for whom a pathological agent was isolated and identified. Of these, 7 patients had Gram-positive infections with different Staphylococcus species. Gram-negative organisms were responsible for 5 of the infections correctly identified by the ${ }^{99 \mathrm{~m}} \mathrm{Tc}$-sulesomab and the remaining patient had a fungal infection. In the other 8 patients, the diagnosis was confirmed by radiology, other blood tests and surgical correlation. There was a single false-positive study, with uptake in a malignant but non-infected pericarditis.

$99 \mathrm{~m}$ Tc-sulesomab showed positive uptake in 11 patients with intra-abdominal infection (fig. 1,2). This was identified correctly despite the fact that there is some ${ }^{99 \mathrm{~m}} \mathrm{Tc}$ sulesomab accumulation in the normal bowel and renal tract. There were 5 intrathoracic sites of infection includ- 
Table 1. Summary of results

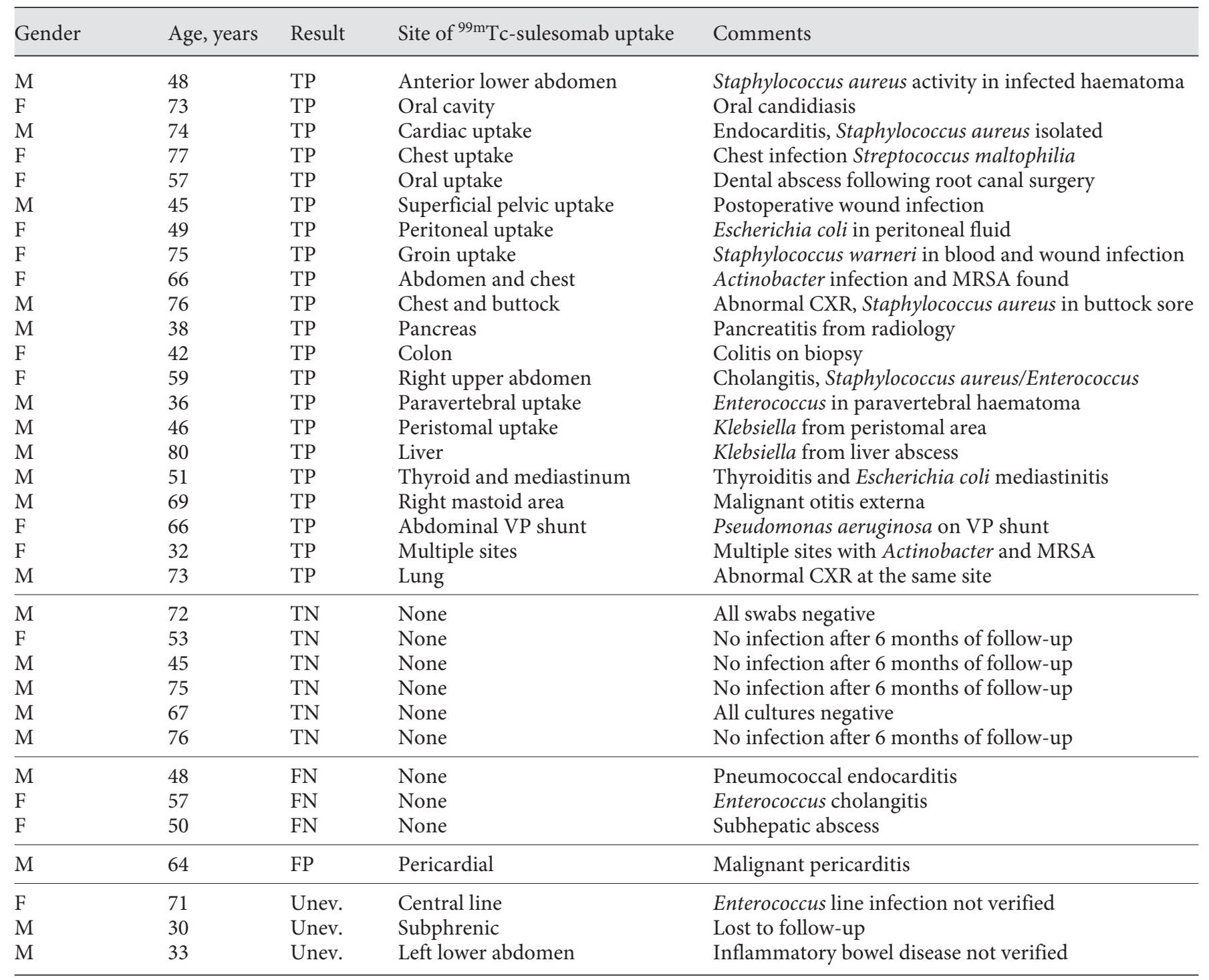

$\mathrm{TP}=$ True-positive; $\mathrm{TN}$ = true-negative; $\mathrm{FP}$ = false-positive; $\mathrm{FN}=$ false-negative; Unev. = unevaluable; $\mathrm{MRSA}=$ methicillin-resistant Staphylococcus aureus; CXR = chest X-ray; VP = ventriculoperitoneal.

ing mediastinitis (fig. 3) and bacterial endocarditis. An infected buttock abscess was also identified (fig. 4). In both the thorax and the abdomen, the use of SPECT, especially in the later 4- to 6-hour image, helped determine that the activity was not physiological and therefore identify the site of infection. ${ }^{99 \mathrm{~m} T c-s u l e s o m a b}$ was also able to identify a case of malignant otitis externa, an infected vascular graft (fig. 5), a dental abscess and oral candidiasis.

There were 6 true-negative studies, in which a negative study was followed up by negative cultures with no clini- cal features to suggest ongoing infection during at least 6 months of follow-up after the scan. There were 3 falsenegative studies. One patient had an Enterococcus septicaemia, the origin of which was presumed to be the gall bladder, but no abnormal uptake of ${ }^{99 \mathrm{~m}} \mathrm{Tc}$-sulesomab was seen at this site. Another patient had a pneumococcal endocarditis, but the ${ }^{99 \mathrm{~m}} \mathrm{Tc}$-sulesomab chest SPECT demonstrated only normal physiological uptake. The third patient had a subhepatic abscess which was not identified by ${ }^{99 \mathrm{~m}} \mathrm{Tc}$-sulesomab but was subsequently correctly lo- 


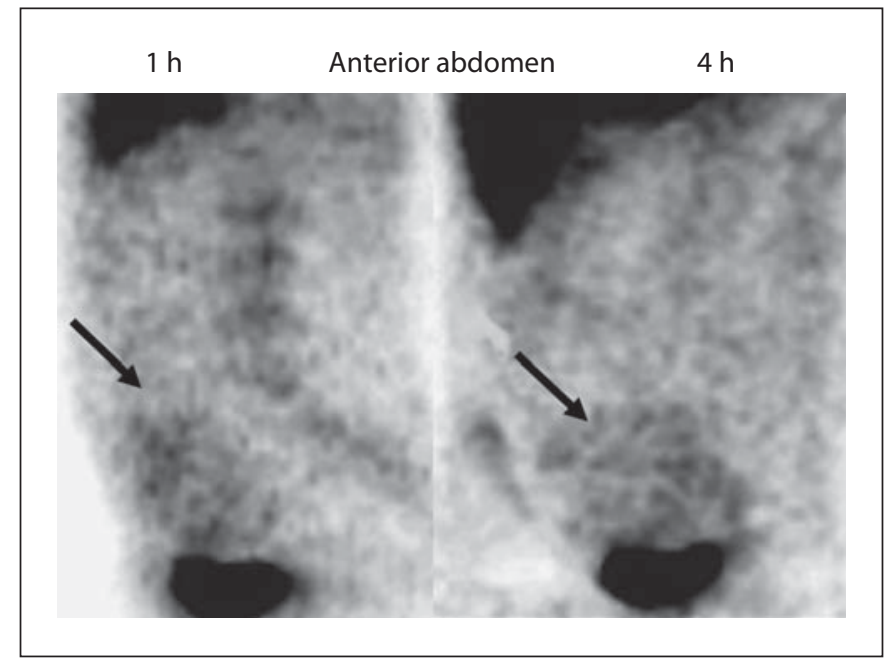

Fig. 1. Increased uptake of ${ }^{99} \mathrm{~m} \mathrm{Tc}$-selusomab within infected haematoma (arrows) with images at 1 and $4 \mathrm{~h}$.
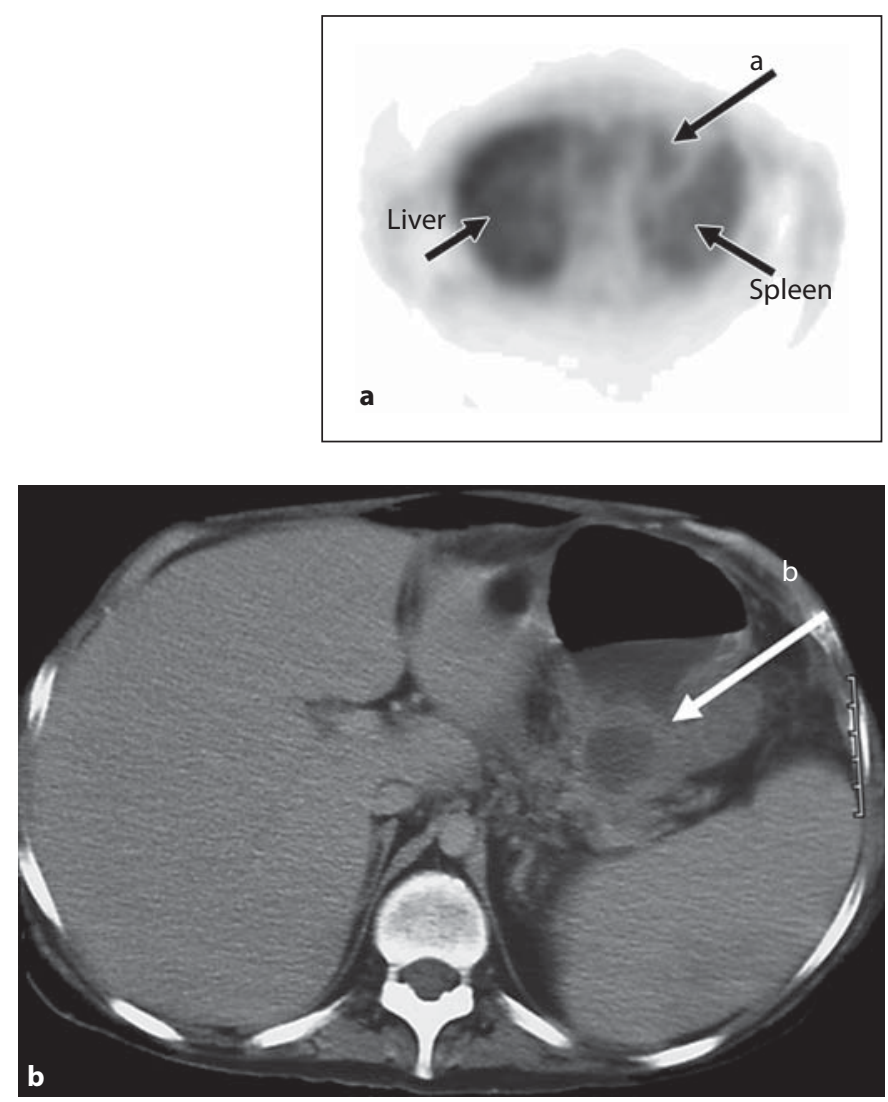

Fig. 2. a Increased tracer uptake in tail of pancreas in a patient with infected pancreatitis (marked 'a' on transverse SPECT slice). b Abscess in tail of pancreas (marked 'b' on CT in the same patient).

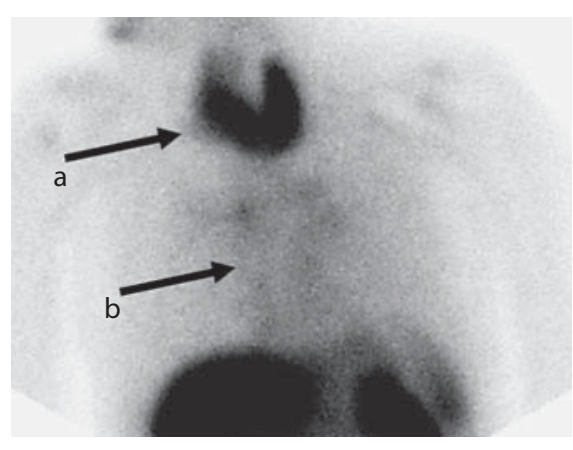

Fig. 3. Increased tracer uptake in patient with infective thyroiditis (arrow 'a') and mediastinitis (arrow 'b').

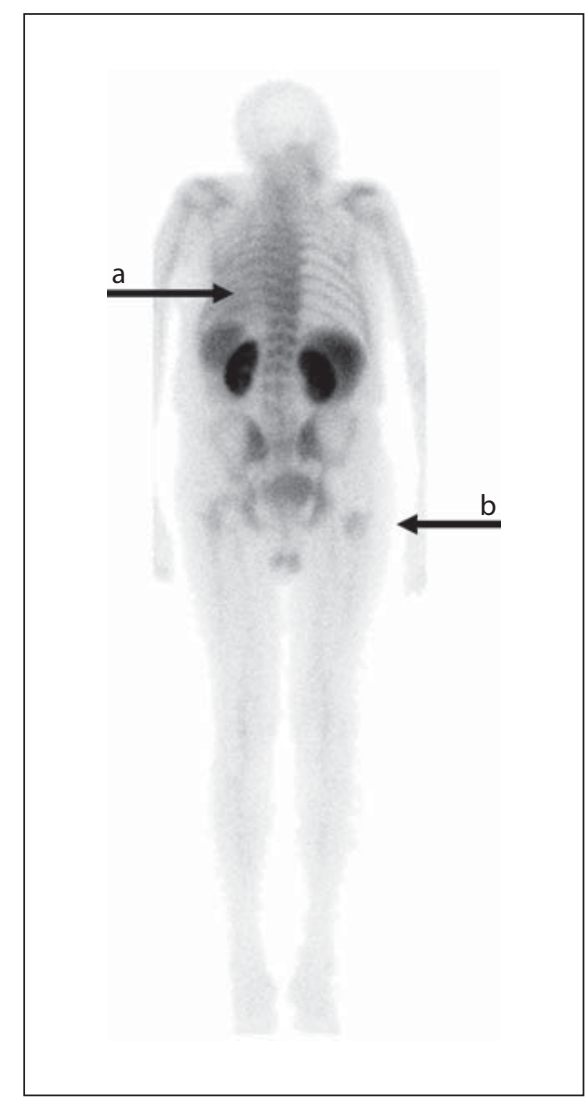

Fig. 4. Increased tracer uptake in left lung (arrow 'a') in a patient with chest X-ray appearances suggestive of infection in the left lung and also in the right buttock with infected skin ulcer (arrow 'b'). 


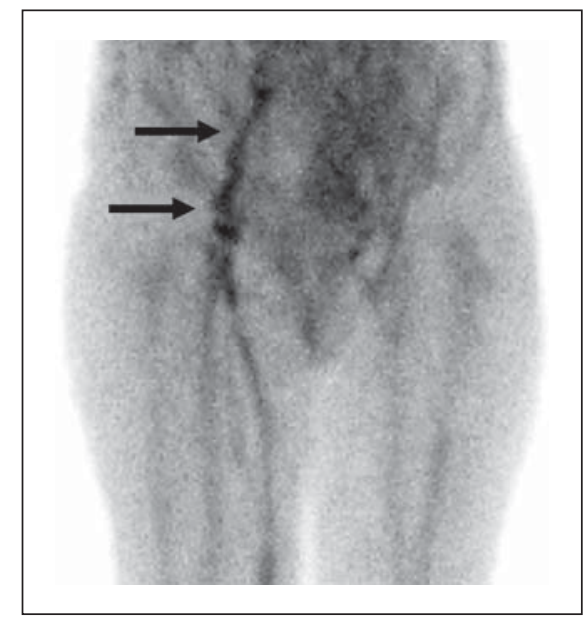

Fig. 5. Increased tracer uptake in infected graft (arrows) on 4-hour image. The unoperated left iliac artery shows normal 'blood pool' activity.

calised using ${ }^{67} \mathrm{Ga}$-citrate imaging. These results gave an overall sensitivity of $88 \%$.

Although the numbers are small, specificity was $86 \%$. A positive predictive value of $95 \%$ and a negative predictive value of $66 \%$ were calculated. The overall accuracy of ${ }^{99 \mathrm{~m}} \mathrm{Tc}$-sulesomab in soft tissue infection was calculated as $87 \%$.

Three patients were unevaluable. In 1 patient, there was uptake in the soft tissue surrounding the site of central line insertion, and although confirmation of an Enterococcus infection was noted on the nuclear medicine request form, the patient's records were missing; therefore, it was not possible to confirm this finding. Another patient was not available for follow-up, and the third patient had a presumed diagnosis of inflammatory bowel disease with caecal uptake of ${ }^{99 \mathrm{~m}} \mathrm{Tc}$-sulesomab, but this could not be confirmed.

\section{Discussion}

This study demonstrates the clinical utility of ${ }^{99 \mathrm{~m} T c-}$ sulesomab as an imaging agent for suspected soft tissue infection. Compared to previous studies for orthopaedic infection, the sensitivity and specificity of ${ }^{99 \mathrm{~m} T c \text {-sule- }}$ somab for soft tissue infection is slightly less than when it is used for identifying bone and joint infections. For bone and joint infection, the sensitivity is $93 \%$ and specificity $89 \%$ [4] compared to a sensitivity of $88 \%$ and a specificity of $86 \%$ for soft tissue infections. However, the small sample size in this study means that an accurate comparison is difficult and this difference may not be significant. In addition, this is a retrospective study, and some caution must be exercised when comparing these results to those of a prospective trial, as there is a bias towards choosing patients with the disease in a retrospective study and it may be that in a full prospective trial, the accuracy of the ${ }^{99 \mathrm{~m}} \mathrm{Tc}$-sulesomab may be less than in our series of patients.

The patients selected in our study were imaged with ${ }^{99 \mathrm{~m}} \mathrm{Tc}$-sulesomab either by serendipity (for example, the suspected infection was in fact within the soft tissues and not bone) or pragmatism (the 'Friday' patient presenting when no pharmacist is available for cell labelling and a prompt result is needed). This will lead to some distortion of the population studied, but the wide range of sites and types of infection identified in our study suggest that there is a fairly eclectic group of clinical situations in which ${ }^{99 \mathrm{~m}}$ Tc-sulesomab may be useful.

A meta-analysis of different imaging techniques for intra-abdominal infections published a sensitivity of $88 \%$ and a specificity of $93 \%$ in 682 patients imaged with ${ }^{111} \mathrm{In}$ labelled white cells, while for ${ }^{99 \mathrm{~m}} \mathrm{Tc}-\mathrm{HMPAO}-$ labelled leucocytes, the combined sensitivity was $88 \%$ and the specificity was $91 \%$ in 1,427 patients [5]. Our figures, though not referring exclusively to abdominal infection, show a similar sensitivity and specificity. Clearly, this would need to be tested in the setting of a formal randomised control trial. There are limitations when comparing a single-site review of 34 patients with such a large meta-analysis, but it does suggest that this agent should be considered for further evaluation as an agent for imaging suspected soft tissue infection, as long as the caveat on positivity (e.g. increasing accumulation over time at a non-physiological site) is maintained.

There are certain clinical advantages in using ${ }^{99 \mathrm{~m}} \mathrm{Tc}-$ sulesomab, as no cell labelling is required and preparation of the product takes only about $30 \mathrm{~min}$. It could be particularly attractive when considering imaging patients in whom cell labelling is not desirable or for those in whom the retrovirus or hepatitis infection status is unknown or positive. It could also be useful in cases where speed of diagnosis is important. This is illustrated in our series for each patient (table 1, fig. 3), where a life-threatening mediastinal infection was identified in a comatose patient who was known be an intravenous drug user, but for whom full counselling for an HIV test (a legal requirement in the UK) was clearly not possible.

When comparing the results from other antibodybased products, such as ${ }^{99 \mathrm{~m}} \mathrm{Tc}$-fanelusomab and ${ }^{99 \mathrm{~m}} \mathrm{Tc}$ - 
BW150/183 [6, 7], the main characteristic of ${ }^{99 \mathrm{~m}} \mathrm{Tc}$-sulesomab is the high persistent blood pool activity, low or minimal bone marrow activity but possibly more liver, bowel and urinary activity. This is probably due to the fact that as a Fab' fragment, there is little immunogenic reaction and as a consequence of this, not much activity is cleared from the blood into the bone marrow and spleen. ${ }^{99 \mathrm{~m}} \mathrm{Tc}$-sulesomab has a low rate $(<5 \%)$ of producing human anti-mouse antibodies [8], and thus repeat imaging, if necessary, could be done safely in the majority of patients.

A full understanding of the mechanism of action of ${ }^{99 \mathrm{~m}} \mathrm{Tc}$-sulesomab should be sought because without this it is impossible to predict those clinical situations where ${ }^{99 \mathrm{~m}} \mathrm{Tc}$-sulesomab may be used and those where it will be inaccurate.

\section{Conclusion}

${ }^{99} \mathrm{~m} \mathrm{Tc}$-sulesomab is able to identify a wide range of soft tissue infections in a variety of clinical situations with a range of causative organisms. The results of this retrospective study indicate sufficient accuracy to justify a full prospective study on the use of ${ }^{99 \mathrm{~m}} \mathrm{Tc}$-sulesomab in presumed soft tissue infection.

\section{References}

1 Ryan PJ: Leukoscan for orthopaedic imaging in clinical practice. Nucl Med Commun 2002;23:707-714.

2 Skehan SJ, White JF, Evans JW, Parry-Jones DR, Solanki CK, Ballinger JR, Chilvers ER, Peters AM: Mechanism of accumulation of ${ }^{99 \mathrm{~m}} \mathrm{Tc}$-sulesomab in inflammation. J Nucl Med 2003;44:11-18.

3 Becker W: Bone and soft tissue; in Cox $\mathrm{PH}$, Buscombe JR (eds): The Imaging of Infection and Inflammation. Amsterdam, Kluwer, 1998, pp 199-217.
4 Hakki S, Harwood SJ, Morissey MA, Camblin JG, Laven DL, Webster WB: Comparative study of monoclonal antibody scan in diagnosing orthopaedic infection. Clin Orthop 1997;335:275-285.

5 Annovazzi AA, Bagni BB, Burroni LC, D’Alessandria CA, Signore AA: Nuclear medicine imaging of inflammatory/infective disorders of the abdomen. Nucl Med Commun 2005;26:657-664.

6 6 Love C, Tronco GG, Palestro CJ: Imaging of infection and inflammation with $99 \mathrm{~m}$ Tcfanolesomab. Q J Nucl Med Mol Imaging 2006;50:113-120.
7 Sciuk J, Brandau W, Vollet B, Stucker R, Erlemann R, Bartenstein P, Peters PE, Schober O: Comparison of technetium-99m polyclonal human immunoglobulin and technetium-99m monoclonal antibodies for imaging chronic osteomyelitis: first clinical results. Eur J Nucl Med 1991;18:401-407.

8 Shanthly N, Aruva MR, Zhang K, Mathew B, Thakur ML: ${ }^{99 \mathrm{~m} T c-F a n o l e s o m a b: ~ a f f i n i t y, ~}$ pharmacokinetics and preliminary evaluation. Q J Nucl Med Mol Imaging 2006;50: 104-112. 